\title{
Variáveis do processo de briquetagem e qualidade de briquetes de biomassa florestal
}

\author{
Thielly Schmidt Furtado ${ }^{1}$, Marina Valin ${ }^{1}$, Martha Andreia Brand ${ }^{1}$, Antonio Francisco Jurado Bellote ${ }^{2}$
}

'Universidade do Estado de Santa Catarina (Udesc), Av. Luís de Camões, 2090, CEP 88035-001, Florianópolis, SC, Brasil, thiellysf@hotmail.com; tonafloresta@gmail.com; a2mab@cav.udesc.br; ${ }^{2}$ Florestas, Estrada da Ribeira Km 11, CP 319, CEP 83411-000, Colombo, PR, Brasil bellote@cnpf.embrapa.br

\begin{abstract}
Resumo - Na busca pelo aproveitamento dos resíduos gerados desde a produção florestal até os processos de transformação industrial da biomassa, desenvolveu-se o processo de briquetagem. A aglomeração de partículas de madeira facilita as operações de manuseio do material combustível, além de concentrar a energia disponível em termos de volume. O objetivo do presente trabalho foi avaliar se a matéria-prima tem influência na qualidade do briquete e verificar o efeito da pressão aplicada durante o processo nas características energéticas e mecânicas do produto final, além de avaliar o comportamento da mistura de materiais (MIX) em relação aos materiais puros. Os briquetes foram produzidos em uma briquetadeira piloto, tipo pistão hidráulico, a $120^{\circ} \mathrm{C}$ com pressão constante de 50 bar por oito minutos e 65,95 ou 130 bar por dois minutos. Foram utilizados seis briquetes para cada tratamento. As características avaliadas foram poder calorífico superior (PCS), densidade aparente e resistência à compressão. A matéria-prima tem maior influência na qualidade dos briquetes do que a pressão de compactação. A baixa pressão é a mais indicada para briquetes de biomassa florestal de Pinus sp. Nesta, o MIX apresentou qualidade satisfatória de briquetes com PCS de $4.773 \mathrm{kcal} \mathrm{kg}^{-1}$, densidade aparente de $1.220 \mathrm{~kg} \mathrm{~m}^{-3}$ e resistência à compressão de $167 \mathrm{kgf} \mathrm{cm}^{-2}$.
\end{abstract}

Termos para indexação: Pinus sp; poder calorífico superior; densidade aparente; resistência à compressão.

\section{Variables of briquetting process and quality of forestry biomass briquettes}

\begin{abstract}
In the quest for recovery of waste generated from forest production to the process of industrial transformation of the biomass it was developed the process of briquetting. The cluster of wood particles facilitates the operations of handling of combustible material in addition to concentrating the available energy in terms of volume. The purpose of this study was to evaluate whether the raw material affects the quality of the briquette and verify the effect of pressure applied during the mechanical and energy characteristics of the final product, and to evaluate the behavior of the material mix (MIX) compared to pure materials. The briquettes were produced in a pilot briquetter, hydraulic piston, $120^{\circ} \mathrm{C}$ with a constant pressure of 50 bar for eight minutes and 65,95 or 130 bar for two minutes. Six briquettes were used for each treatment. The characteristics evaluated were calorific value (GCV), bulk density and compressive strength. The raw material has a greater influence on the quality of briquettes than the compaction pressure. The low pressure is the most suitable for Pinus sp forest biomass briquettes. In this, MIX submitted satisfactory quality of briquettes with PCS $4,773 \mathrm{kcal} \mathrm{kg}^{-1}$, density $1220 \mathrm{~kg} \mathrm{~m}^{-3}$ and compressive strength of $167 \mathrm{kgf} \mathrm{cm}^{-2}$.
\end{abstract}

Index terms: Pinus sp., gross calorific value, density, compressive strength.

\section{Introdução}

A indústria de base florestal, como um todo, tem por característica, a grande geração de resíduos ao longo do processo de produção. Segundo Boundelle et al. (2002), o setor madeireiro apresenta um grande potencial para o aproveitamento de resíduos, já que as perdas são inerentes ao processo produtivo, representando $40 \%$ a $70 \%$ do volume de matéria-prima sem uma utilização determinada. Estes resíduos particulados são obtidos durante a colheita e beneficiamento da madeira e, por muito tempo, não tiveram destinação adequada. Recentemente, passou-se a utilizá-los como combustível, entretanto, o uso do material particulado apresenta desvantagens, como a exigência de adequação das caldeiras para uso de finos e dificuldades de transporte (Dias, 2002).

A necessidade de recuperar finos de carvão oriundos do processo de beneficiamento de minérios fez com que surgisse a técnica de aglomeração denominada 
briquetagem. Em 1848, Easby desenvolveu um processo que possibilitava a formação de aglomerados sólidos de tamanho e forma variados, a partir de frações finas de qualquer tipo de carvão, por meio da pressão exercida sobre esse material (Carvalho \& Brinck, 2004).

A briquetagem é um processo de reconstrução, ou seja, é a reconsolidação de material particulado por meio da aplicação de temperatura e pressão a uma massa de partículas, com ou sem adição de ligantes (Quirino \& Brito, 1991). No caso da madeira, esta adição não é necessária, pois devido à alta temperatura aplicada, a lignina sofre um processo denominado transição vítrea, atuando como agente de ligação entre as partículas, além de criar uma camada que protege o briquete de variações de umidade. Segundo Figueiroa \& Moraes (2009), durante o aquecimento da madeira, a lignina torna-se uma cadeia polimérica mais desordenada e móvel, pois é um polímero termoplástico. Entretanto, após a secagem do briquete, ela volta e ser rígida e apresenta um aspecto vitrificado num processo denominado transição vítrea que é a passagem de um estado desordenado rígido (vítreo) do polímero para um estado desordenado maior, no qual as cadeias poliméricas possuem uma maior mobilidade.

$\mathrm{O}$ uso de briquetes em caldeiras industriais elimina a necessidade de adequação das mesmas, permitindo também uma queima mais uniforme e melhoria das condições de transporte, manuseio, armazenamento e alimentação das caldeiras (Dias, 2002). Outra vantagem do uso de briquetes é a concentração da energia disponível, ou seja, há maior geração de energia por unidade de massa do material, fato que também contribui para a economia no transporte, pois um mesmo volume de briquetes pode ter cinco vezes mais energia que a madeira in natura (Quirino \& Brito, 1991).

Atualmente, devido às pressões ambientais por otimização no uso de recursos naturais, busca-se desenvolver metodologias para a briquetagem de resíduos florestais ou da indústria madeireira.

As características que qualificam um briquete são: poder calorífico superior (PCS); densidade; e resistência à compressão. Estas características são influenciadas por variáveis relacionadas ao processo de produção. O poder calorífico superior indica o potencial de produção de energia por unidade de massa; a densidade, expressa a quantidade de material por unidade de volume, portanto, quanto maior, mais concentrada está a energia e, assim, tem-se um briquete com maior potencial energético em um mesmo volume; a resistência máxima à compressão é um parâmetro para a definição da resistência do briquete durante o transporte e o armazenamento.

Assim, o objetivo do presente trabalho foi avaliar a hipótese de que a matéria-prima tem influência na qualidade do briquete e verificar o efeito da pressão aplicada durante o processo nas características energéticas e mecânicas do produto final, além de avaliar a qualidade da mistura dos diferentes materiais disponíveis, em comparação ao uso de materiais puros.

\section{Material e métodos}

O material utilizado para a confecção dos briquetes foi obtido de uma cogeradora instalada na cidade de Lages, SC. A cogeradora adquire de empresas florestais e madeireiras da região os resíduos do processo produtivo e os utiliza como combustível para a geração de energia térmica e elétrica. As matérias-primas utilizadas com maior frequência para a produção dos briquetes foram casca de Pinus sp., cavaco de Pinus sp., mistura de diferentes matérias-primas (MIX) e serragem de Pinus sp. É importante salientar que o controle da mistura é uma informação restrita, sendo determinada pela cogeradora e enviada para análise.

As amostras foram analisadas no Centro de Ciências Exatas e Tecnológicas da Universidade do Planalto Catarinense-CCET/Uniplac. O material foi processado em moinho de martelo para a obtenção de partículas de, no máximo, $1,5 \mathrm{~cm}$ de comprimento. Em seguida, foram acondicionados em câmara climatizada até atingirem umidade em torno de $9 \%$, aferida em termobalança.

Foram confeccionados seis briquetes para cada tratamento, em briquetadeira piloto tipo pistão hidráulico, à temperatura constante de $120^{\circ} \mathrm{C}$, durante dez minutos, nos oito primeiros minutos à pressão de 50 bar, e à pressão desejada ( 65 bar - baixa, 95 bar - média ou 130 bar - alta) durante os últimos dois minutos. Os valores de pressão utilizados no experimento indicam o uso de baixa, média e alta pressão para a produção dos briquetes.

$\mathrm{O}$ procedimento adotado para a briquetagem foi desenvolvido através da parceria entre os pesquisadores dos Laboratórios de Energia de Biomassa da Uniplac e Laboratório de Produtos Florestais do Ministério do Meio Ambiente. O equipamento da Uniplac foi o primeiro produzido no Brasil para testes laboratoriais em escala piloto com a reprodução das condições de 
briquetadeiras industriais. No entanto, em função da escala da produção, foi necessário o desenvolvimento da metodologia acima descrita que permite a reprodução do que acontece no equipamento industrial.

Assim, o tempo inicial a uma pressão menor reproduz o tempo que o material entra no equipamento industrial, sofrendo aquecimento e sendo conduzido para a parte final da briquetadeira, onde ocorre a consolidação do briquete. Nesta etapa, o material é aquecido, mas não sofre pressão suficiente para promover a ligação interna do material lignocelulósico.

Portanto, a pressão inicial, mais baixa, simula a alimentação do material na briquetadeira, onde ocorre o aquecimento da biomassa e consequente início do processo de transição vítrea. Os minutos finais de ensaio, com elevação da pressão, simulam a passagem da biomassa, já aquecida pelo cone final das briquetadeiras industriais, onde ocorre a ligação interna e consolidação do briquete, com posterior saída do mesmo da briquetadeira.

Os parâmetros de análise foram determinados após a confecção dos briquetes. A densidade foi determinada por meio de pesagem em balança de precisão e medição com paquímetro de diâmetro e altura de cada briquete. O poder calorífico foi aferido segundo a norma DIN 51900, em bomba calorimétrica. O teste de resistência à compressão foi realizado em máquina universal de ensaios, segundo protocolo desenvolvido especificamente para briquetes de madeira, utilizando o prato para compressão de $100 \mathrm{kN}$, com velocidade de $2 \mathrm{~mm} \mathrm{~min}^{-1}$, de modo a se obter a força máxima suportada pelo briquete até o rompimento. Os resultados serão apresentados em $\mathrm{kgf} \mathrm{cm}^{-2}$, considerando-se para o cálculo a área de contato do briquete com o prato ( $5 \mathrm{~cm}^{2}$, em média).

O delineamento experimental utilizado foi completamente casualizado, em esquema fatorial (4 matérias-primas x 3 pressões), com 6 repetições por tratamento. As análises estatísticas foram realizadas com auxílio do software SAS (Statistical Analysis System, 2002).

\section{Resultados e discussão}

A Tabela 1 demonstra que os fatores material, pressão e a interação material, e pressão tiveram influência significativa sobre o PCS e densidade aparente dos briquetes pelo teste F. Deste modo, os testes de médias foram realizados considerando o material de origem dentro de cada pressão e a pressão dentro de cada material de origem. Já a resistência à compressão sofreu influência apenas do material.

Tabela 1. Resumo da análise de variância global para poder calorífico superior (PCS), densidade aparente e resistência à compressão em função do material de origem e pressão aplicada.

\begin{tabular}{lrrrr}
\hline \multirow{2}{*}{ FV } & GL & \multicolumn{1}{c}{ PCS } & Densidade & $\begin{array}{c}\text { Resistência à } \\
\text { compressão }\end{array}$ \\
\cline { 3 - 5 } & & \multicolumn{1}{c}{ F } & \multicolumn{1}{c}{ F } & \multicolumn{1}{c}{ F } \\
\hline Matéria-prima (M) & 3 & $581,32^{*}$ & $154,67 *$ & $107,56^{*}$ \\
Pressão (P) & 2 & $4,63^{*}$ & $12,35^{*}$ & $2,14^{\mathrm{NS}}$ \\
Interação MxP & 6 & $13,25^{*}$ & $5,72^{*}$ & $1,37^{\mathrm{NS}}$ \\
CV (\%) & & & & \\
\hline
\end{tabular}

* significativo a $5 \%$ de probabilidade de erro pelo teste $\mathrm{F}$; ${ }^{\text {NS }}$ Não significativo

Analisando o comportamento dos diferentes materiais dentro de cada pressão (Tabela 2), verificou-se que os cavacos apresentaram os melhores resultados em todas as pressões analisadas. A serragem equiparou-se aos cavacos nas pressões baixa e média, com o MIX ocupando a posição intermediária.

A briquetagem de diferentes tipos de biomassa, florestal e agrícola, tem demonstrado que em materiais com maior conteúdo de extrativos voláteis, o aumento da pressão exerce efeito contrário sobre o poder calorífico dos briquetes, reduzindo-o devido à perda dos voláteis, que é consequência da temperatura do processo aliado ao aumento da pressão, causando o escape destes compostos para o meio, devido à redução dos espaços vazios dentro do briquete. Isto pode ser verificado no componente casca, que possui maior quantidade de extrativos (Vital et al., 1989) em relação aos demais materiais, e que teve redução do poder calorífico com o aumento da pressão.

Tabela 2. Estudo dos efeitos simples dos fatores matéria-prima e pressão sobre a variável poder calorífico superior (PCS).

\begin{tabular}{lccc}
\hline \multirow{4}{*}{ Matéria-prima a } & \multicolumn{3}{c}{ Pressão } \\
\cline { 2 - 4 } & Baixa & Média & Alta \\
\hline Casca & A 4223 c & B 3874 c & B 3864 c \\
Cavaco & A 5022 a & A $5031 \mathrm{a}$ & A 5077 a \\
MIX & B 4773 b & AB 4826 b & A 4957 ab \\
Serragem & A 4968 a & A 4962 ab & A 4884 b
\end{tabular}

Letras maiúsculas na linha comparam as pressões dentro de cada material a $5 \%$ de probabilidade pelo teste de Tukey; Letras minúsculas na coluna comparam os materiais dentro de cada pressão a $5 \%$ de probabilidade pelo teste de Tukey. 
A casca apresentou maior PCS na menor pressão, pois tem elevada quantidade de substâncias voláteis com poder calorífico alto. O comportamento de maior perda de voláteis em função do aumento da pressão é confirmado por Cataluña \& Silva (2006).

Além do efeito da pressão, a casca apresentou menor PCS em todas as situações devido, possivelmente, ao seu elevado teor de cinzas. Este material é oriundo de processo industrial e, portanto, a contaminação por substâncias inorgânicas é alta em decorrência do contato direto com solo e outras superfícies. O teor de cinzas da casca gira em torno de $14 \%$, enquanto os demais materiais apresentam, no máximo, 4\% (Furtado et al., 2009).

O teor de cinzas representa a fração inerte da amostra, a qual é incombustível. Quando este apresenta valores elevados, reduz-se o PCS, que possui relação direta com a composição química da matéria-prima queimada.

A casca tem o menor PCS, enquanto cavaco e serragem apresentam valores mais elevados, portanto, a mistura (MIX) ficou em posição intermediária nas três pressões. O maior PCS absoluto foi verificado na pressão de 130 bar, porém não apresentou diferença significativa quando comparada à pressão de 95 bar que, por sua vez, não diferiu estatisticamente da pressão de 65 bar. É importante salientar que não há controle da mistura neste experimento, pois esta é uma informação estratégica de posse da empresa fornecedora dos materiais. Os materiais cavaco e serragem apresentaram os maiores valores de PCS (Tabela 2).

De acordo com o material usado, há uma pressão mais indicada para se obter melhores resultados em termos de PCS. Entretanto, pode-se indicar, considerando apenas a variável PCS o uso da baixa pressão, pois demanda um menor dispêndio de energia com resultados satisfatórios.

O PCS do MIX só é inferior ao do cavaco, o que demonstra a qualidade energética dessa matéria-prima. $\mathrm{O}$ cavaco puro dificilmente será utilizado para briquetagem, pois pode ser destinado a fins economicamente mais rentáveis, como celulose e papel.

Estudando os efeitos do material dentro de cada pressão, os briquetes de casca de Pinus sp. apresentaram a maior densidade aparente em todas as pressões estudadas, enquanto os demais materiais não apresentaram diferenças significativas entre si para a variável densidade final de briquete (Tabela 3 ).
Tabela 3. Estudo dos efeitos simples dos fatores matéria-prima e pressão sobre a variável densidade.

Média das densidades (kg.m-3)

\begin{tabular}{lccc}
\multirow{2}{*}{ Matéria-prima } & \multicolumn{3}{c}{ Pressão } \\
\cline { 2 - 4 } & Baixa & Média & Alta \\
\hline Casca & B $1330 \mathrm{a}$ & AB $1390 \mathrm{a}$ & A $1410 \mathrm{a}$ \\
Cavaco & B $1190 \mathrm{~b}$ & AB $1220 \mathrm{~b}$ & A $1260 \mathrm{~b}$ \\
MIX & A $1220 \mathrm{~b}$ & A $1210 \mathrm{~b}$ & A $1190 \mathrm{bc}$ \\
Serragem & A $1230 \mathrm{~b}$ & A $1240 \mathrm{~b}$ & A $1260 \mathrm{~b}$ \\
\hline
\end{tabular}

Letras maiúsculas na linha comparam as pressões dentro de cada material a 5\% de probabilidade pelo teste de Tukey; Letras minúsculas na coluna comparam os materiais dentro de cada pressão a $5 \%$ de probabilidade pelo teste de Tukey.

Estudo realizado por Mendes (1993) com árvores de Pinus taeda com idades entre 8 e 20 anos demonstra que a casca possui menor densidade básica em relação à madeira. Enquanto a densidade básica da madeira varia de $332 \mathrm{~kg} \mathrm{~m}^{-3}$ a $396 \mathrm{~kg} \mathrm{~m}^{-3}$, a da casca apresenta valores entre $255 \mathrm{~kg} \mathrm{~m}^{-3}$ e $301 \mathrm{~kg} \mathrm{~m}^{-3}$. Deste modo, consegue-se obter um briquete mais denso, segundo o princípio da razão de compactação aplicado na produção de painéis. Quanto menor a densidade do material de origem, maior a densidade aparente do produto final após aplicação de pressão para compactação (Iwakiri et al., 2008).

$\mathrm{O}$ aumento da pressão de compactação não teve resultado significativamente superior na densidade final do briquete.

Para casca, o aumento da pressão, mesmo elevando a densidade, resultou em redução de quantidade de energia gerada. Isto pode ser verificado pela multiplicação do PCS pela densidade, em que na menor pressão obtém-se 5.617 $\mathrm{Mcal} \mathrm{m}^{-3}$ e $5.448 \mathrm{Mcal} \mathrm{m}^{-3}$ na maior.

É possível verificar o ganho de aproximadamente quatro vezes, proporcionado pelos briquetes em termos de quantidade de material por unidade de volume em comparação com a madeira in natura (Tabela 3), semelhante ao observado por Mendes (1993). Isso contribui expressivamente para a redução dos custos de transporte do material combustível.

Na Tabela 4 são apresentados os valores da resistência à compressão, ou seja, qual a força máxima suportada pelo briquete até o seu rompimento no teste de compressão. A serragem apresentou menores valores de resistência à compressão. Isto pode ser explicado pelo fato de ter maior proporção de partículas finas, as quais não apresentam boas propriedades mecânicas. 
O MIX, por ser uma mistura de diversos tipos de materiais com dimensões diferenciadas, é um material mais heterogêneo e, portanto, com maior dificuldade de acomodação das partículas, o que leva à existência de mais espaços vazios e resulta na menor resistência do briquete.

Iwakiri et al. (2008), em estudo com painéis de madeira, verificaram que partículas mais alongadas e com menor densidade inicial apresentam maior razão de compactação e, consequentemente, valores maiores de densidade e resistência à compressão. Isto explica a maior resistência à compressão da casca.

Tabela 4. Estudo dos efeitos simples do fator matéria prima sobre a variável resistência à compressão.

\begin{tabular}{lc}
\hline Matéria-prima & $\begin{array}{c}\text { Média da resistência à compressão } \\
\left(\mathbf{k g f} \mathbf{~ c m}^{-2}\right)\end{array}$ \\
\hline Casca & $192 \mathrm{a}$ \\
Cavaco & $183 \mathrm{a}$ \\
Serragem & $115 \mathrm{c}$ \\
MIX & $167 \mathrm{~b}$ \\
\hline
\end{tabular}

Letras iguais indicam valores significativamente iguais no teste de Tukey a $5 \%$.

Quanto ao cavaco, a maior proporção de fibras inteiras em relação à serragem e MIX é responsável pela resistência à compressão elevada. Durante a produção dos cavacos, busca-se obter material com a maior quantidade de fibras intactas possível, enquanto a serragem é um rejeito e não tem um padrão de qualidade bem definido. Como o MIX, a mistura de todos os materiais apresenta características intermediárias.

Com os resultados obtidos, é possível afirmar que a baixa pressão é a mais indicada para a produção de briquetes de biomassa florestal de Pinus sp., pois há menor dispêndio de energia para operar a briquetadeira e, as propriedades dos briquetes apresentam-se satisfatórias para qualquer dos materiais. Os resultados também demonstram que as propriedades mecânicas iniciais da matéria-prima influenciam a qualidade final do briquete.

Brito \& Nucci (1984), trabalhando com briquetagem de carvão de Pinus oocarpa, P. caribaea var. hondurensis e P. caribaea var. bahamensis, observarm que as pressões de compactação não influem decisivamente na qualidade dos briquetes. Entretanto, indicaram a utilização de $300 \mathrm{~kg} \mathrm{~cm}^{-2}$ ou 294 bar, provavelmente, por estarem trabalhando com carvão, material que exige a adição de ligantes e, portanto, maior pressão para promover a adesão entre os componentes, o que não ocorre com a madeira in natura.

A casca possui alta disponibilidade, porém, quando pura, apresenta qualidade inferior; o cavaco possui as melhores características, entretanto tem outra destinação. Neste cenário, o MIX é uma opção de combustível para a cogeração em situações de baixa disponibilidade do material puro, sem perdas na qualidade final dos briquetes.

\section{Conclusões}

- A matéria-prima tem maior influência sobre a qualidade dos briquetes do que as variáveis do processo;

- A baixa pressão é indicada para a produção de briquetes de biomassa florestal de Pinus sp.;

- O MIX pode ser usado sem prejuízos na qualidade do briquete.

\section{Referências}

BOUNDELLE, G. M.; CHIES, D.; MARTINS, D. G. O processo de fabricação de painéis compensados no Estado do Paraná analisado por meio dos rendimentos e dos resíduos gerados. In: CONGRESSO IBERO-AMERICANO DE PESQUISA E DESENVOLVIMENTO DE PRODUTOS FLORESTAIS, 2., 2002, Curitiba. Anais. Curitiba: UFPR, 2002. Disponível em: $<$ http://www.remade.com.br >. Acesso em: 27 abr. 2010.

BRITO, J. O.; NUCCI, O. de. Estudo tecnológico da madeira de Pinus spp para a produção de carvão vegetal e briquetagem. IPEF, n. 26, p. 25-30, abr. 1984. Disponível em: <www.ipef.br/ publicacoes/scientia/nr26/cap04.pdf>. Acesso em: 30 abr. 2010.

CARVALHO, E. A. de; BRINCK, V. Briquetagem em Tratamento de Minérios. In: LUZ, A. B.; SAMPAIO, J. A.; ALMEIDA, S.

L. M. (Eds). Tratamento de Minérios. $4^{\mathrm{a}}$ ed. Rio de Janeiro: CETEM/MCT, 2004, p.613-635.

CATALUÑA, R.; SILVA, R. Desenvolvimento de um equipamento para avaliação do efeito do etanol na pressão de vapor e entalpia de vaporização em gasolinas automotivas Química Nova, São Paulo, v. 29, n. 3, mai.jun, 2006. Disponível em: <http://www.scielo.br/scielo.php?script=sci_arttext\&pid $=\mathrm{S} 0100-40422006000300029>$. Acesso em: 29 mar. 2010.

DIN 51900: 1-3 - Testing of solid and liquid fuels . Determining the gross calorific value of solid and liquid fuels using the bomb calorimeter, and calculation of net calorific value - Part 1-3. Deutsches Isntitut für Normung e V. Beuth Verlag GmbH, Berlin, 2000.

DIAS, J. J. M. Utilização da biomassa: avaliação dos resíduos e utilização de pellets em caldeiras domésticas. 2002. $102 \mathrm{f}$. Dissertação (Mestrado em Engenharia Mecânica) - Universidade Técnica de Lisboa, Instituto Superior Técnico. Disponível em: $<$ http://purl.pt/6611>. Acesso em: 30 abr. 2010. 
FIGUEIROA, M. J. M.; MORAES, P. D. de. Comportamento da madeira a temperaturas elevadas Ambiente Construído, v. 9, n. 4, p. 157-174, out./dez. 2009. Disponível em: <http://www.seer.ufrgs. br/index.php/ambienteconstruido/article/viewFile/9547/7050>. Acesso em: 30 abr. 2010.

FURTADO, T. S.; FERREIRA, J. C.; NEVES, M. D.; BRAND, M. A. Influência da idade da árvore na eficiência energética dos resíduos de Pinus taeda. In: CONGRESSO BRASILEIRO SOBRE FLORESTAS ENERGÉTICAS, 1., 2009, Belo Horizonte. Anais. Colombo: Embrapa Florestas, 2009. 1 CDROM. (Embrapa Florestas. Documentos, 178). Seção: Matéria Prima e Processos de Conversão Energética.

IWAKIRI, S, ALBUQUERQUE C. E. C. de, PRATA, J. G., COSTA, A. C. B. Utilizaçao de madeiras de Eucalyptus grandis e Eucalyptus dunni para produção de painéis de partículas orientadas - OSB. Ciência Florestal, v. 18, n. 2, p. 265-270, abr./ jun. 2008. Disponível em: <www.ufsm.br/cienciaflorestal/artigos/ v18n2/A12V18N2.pdf>. Acesso em: 27 abr. 2010.
MENDES, C. J. Influência da idade em Pinus taeda sobre a qualidade de madeira para produção de celulose. Série Técnica IPEF, v. 9, n. 27, p. 81-90, Ago.1993. Disponível em: <www. ipef.br/publicacoes/stecnica/nr27/cap07.pdf $>$. Acesso em: 27 abr. 2010.

QUIRINO, W. F.; BRITO, J. O. Características e índice de combustão de briquetes de carvão vegetal. Brasília,DF: IBAMA, Laboratório de Produtos Florestais, 1991. 16 p. (IBAMA-LPF. Série técnica, n. 13).

STATISTICAL ANALYSIS SYSTEM. SAS: Statistical Analysis System - Getting Started with the SAS ${ }^{\circledR}$ Learning Edition. SAS Institute Inc., Cary, USA, 2002. 86 p. Disponível em: $<$ http:// www.sas.com/software/sas9/>. Acesso em: 27 abr. 2010.

VITAL, B. R.; ANDRADE, A. M. de; VALENTE, O. F.; CAMPOS, J. C. Influência da casca no rendimento e na qualidade do carvão vegetal de Eucalyptus grandis. IPEF, n. 41/42, p. 4449, jan./dez. 1989.

Recebido em 22 de maio de 2010 e aprovado em 26 de outubro de 2010 